Article

\title{
Resistive Switching Behavior of Magnesium Zirconia Nickel Nanorods
}

\author{
Tzu-Han Su, Ke-Jing Lee, Li-Wen Wang, Yu-Chi Chang ${ }^{\circledR}$ and Yeong-Her Wang *
}

Institute of Microelectronics, Department of Electrical Engineering, National Cheng-Kung University, Tainan 701, Taiwan; s0964348@hotmail.com (T.-H.S.); Luke.k.j.lee@gmail.com (K.-J.L.); jk220052@gmail.com (L.-W.W.); christina780712@gmail.com (Y.-C.C.)

* Correspondence: yhw@ee.ncku.edu.tw; Tel.: +886-6-275-7575-62352; Fax: +886-6-2080598

Received: 18 May 2020; Accepted: 10 June 2020; Published: 17 June 2020

\begin{abstract}
To effectively improve the uniformity of switching behavior in resistive switching devices, this study developed magnesium zirconia nickel (MZN) nanorods grown on ITO electrodes through hydrothermal method. The field emission scanning electron microscope image shows the NR formation. Al/MZN NR/ITO structure exhibits forming-free and bipolar resistive switching behaviors. MZN NRs have relatively higher ON/OFF ratio and better uniformity compared with MZN thin film. The superior properties of MZN NRs can be attributed to its distinct geometry, which leads to the formation of straight and extensible conducting filaments along the direction of MZN NR. The results suggest the possibility of developing sol-gel NR-based resistive memory devices.
\end{abstract}

Keywords: bipolar; memory; nanorod; resistive; hydrothermal method

\section{Introduction}

Resistive random access memory (RRAM) is one of the strong candidates of next-generation nonvolatile memories (NVM) due to its simple metal/insulator/metal structure, low power consumption, fast switching speed, simple fabrication, and high scalability [1-3]. Resistive switching characteristics have been demonstrated in a variety of materials, including binary metal oxides, chalcogenide materials, and perovskite oxides. Perovskite oxides have attracted much attention due to its simple fabrication, low cost, and controllable process [4,5].

$\mathrm{ZrO}_{2}$ is a good and well-studied ceramic material that exists in three crystalline phases, namely monoclinic, tetragonal, and cubic phases, at different temperatures. $\mathrm{Mg}^{2+}$ ions in $\mathrm{Mg}: \mathrm{ZrO}_{2}$ with lower valance state cause vacancy in the sub-lattice of oxygen, which improves the performance during volume expansion of the phase transformation. Acetylacetone in Ni (II) acetylacetone possesses two pairs of bidentate ligands (BLs) and offers extremely low polymerization rate, resulting in the smoother surface of MZN films. The smoother surface may have fewer defects and low high-resistance switching (HRS) current. In addition, this strong ligand contributes to stability due to the strong bond between $\mathrm{BL}$ and $\mathrm{Zr}[6,7]$. However, local conduction filament in thin films is diverse in each switching, leading to the non-uniform distribution of switching voltages and current distributions which are crucial to the memory array application. Reducing the dimension of devices and achieving a high packing density are also important [8].

In comparison with thin films, nanostructure-based RRAM devices offer structural advantages that are expected to improve the uniformity of memory devices $[9,10]$. The distinct geometry of nanorods (NRs) can control the distribution of oxygen vacancies due to the formation of straight and extensible conducting filaments along the direction of each NRs, thereby improving the uniformity of memory devices [9,11]. In such devices, nanostructure materials, such as GZO NRs [12], NiO/Pt nanowires [13], and $\mathrm{Ni}-\mathrm{NiO}$ core-shell nanowires [14], have been reported to show resistive switching 
characteristics with filament formation and current flow. Although these nanostructure materials show resistive switching behavior, they demand the forming process and their ON/OFF ratio is not good. In addition, nanostructure materials are challenging to grow.

In this work, bipolar resistive switching and forming-free MZN NR memory devices were prepared by hydrothermal method. This method has attracted considerable attention due to its simple procedure, low cost, low temperature, high yield, and controllable process [15-17]. MZN NRs can control oxygen vacancy distribution because injected electrons will be trapped by uniform ionized oxygen vacancies distributed on the MZN NR sidewall. MZN NR memory devices can reduce the coefficient of variation (CV) of ILRS/IHRS from 50\%/90\% to 30\%/50\%. In addition, MZN NR-based RRAM device exhibits good retention up to $10^{4} \mathrm{~s}$ and high ON/OFF ratio of more than $10^{6}$.

\section{Results}

Figure 1 shows the X-ray photoelectron spectroscopy (XPS) spectra of the MZN thin film. The atomic percentage of elements magnesium, zirconate, nickel, and oxygen in MZN thin films verified by XP spectra is $9.2 \%, 21.2 \%, 21.2 \%$, and $69.6 \%$, respectively.

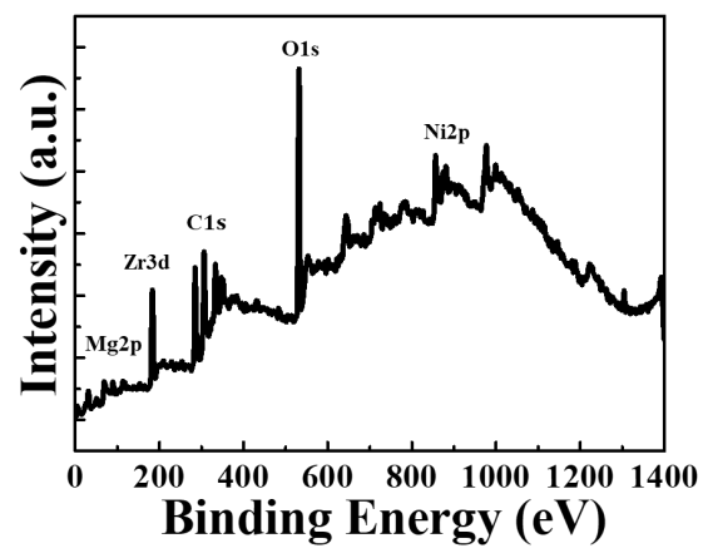

Figure 1. XPS spectra of O 1s, Ni 2p3, Mg 2p, Zr 3d, and C 1s.

Scanning electron microscope (SEM) image shows the formation of MZN NRs on the MZN/ITO structure. Figure 2a shows the smooth surface morphology of the MZN thin film. Scattered MZN NRs were clearly observed on the surface of the MZN thin film soaked for $23 \mathrm{~h}$ (Figure $2 \mathrm{~b}$ ). The shape of the MZN NR is cuboid with width of about 200 (Figure 2c).
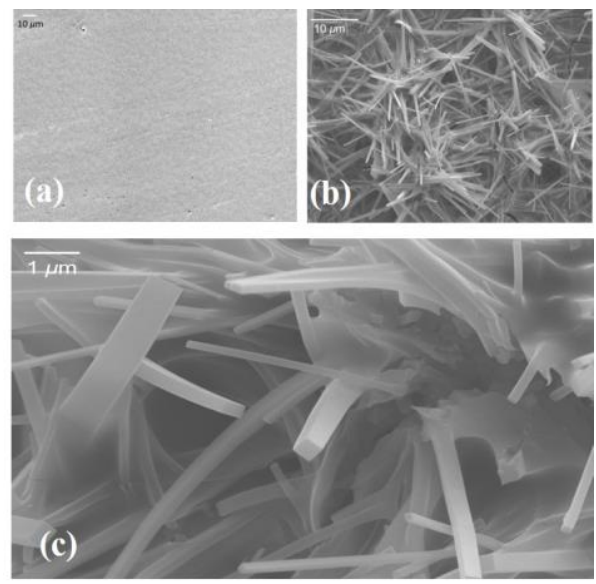

Figure 2. (a) SEM image of the surface morphologies of MZN thin film. (b) Plan-view SEM data of MZN NR nucleation layer that was formed in situ on an ITO surface from the decomposition of the MZN precursor at $60^{\circ} \mathrm{C}$. MZN NRs were grown for $23 \mathrm{~h}$. (c) High-resolution SEM image of MZN NRs. 
Metal chemical components on MZN NR thin film were determined by energy dispersive spectroscopy (EDS, Figure 3). Figure 3a shows the testing location in the MZN NR thin film. Figure 3b and Table 1 show that the chemical composition of MZN NR thin film includes $\mathrm{Mg}=3.15 \%, \mathrm{Zr}=2.15 \%$, $\mathrm{Ni}=5.38 \%, \mathrm{C}=49.13 \%$, and $\mathrm{O}=40.2 \%$.
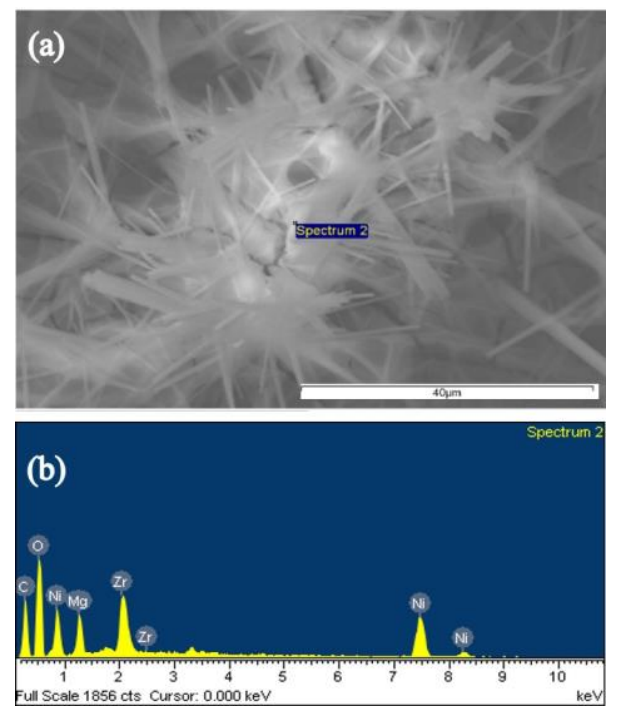

Figure 3. EDS analysis of MZN NR-based thin film (a) testing location and (b) chemical composition.

Table 1. Atomic percentage of MZN NR-based thin films.

\begin{tabular}{ccccccc}
\hline Element & $\mathbf{M g}$ & $\mathbf{Z r}$ & $\mathbf{N i}$ & $\mathbf{C}$ & $\mathbf{O}$ & Totals \\
\hline Atomic $(\%)$ & 3.15 & 2.15 & 5.38 & 49.13 & 40.20 & $100 \%$ \\
\hline
\end{tabular}

The X-ray diffraction analysis of the MZN NR thin film/glass is depicted in Figure 4. No obvious peak was observed in the XRD spectrum of MZN NR/glass. The MZN NR thin film prepared in this experiment is amorphous phase. Compared with polycrystalline phase, amorphous phase is preferred for dielectric layer material because the former may have a high-grain boundary leakage current and lead to a rough film surface [18].

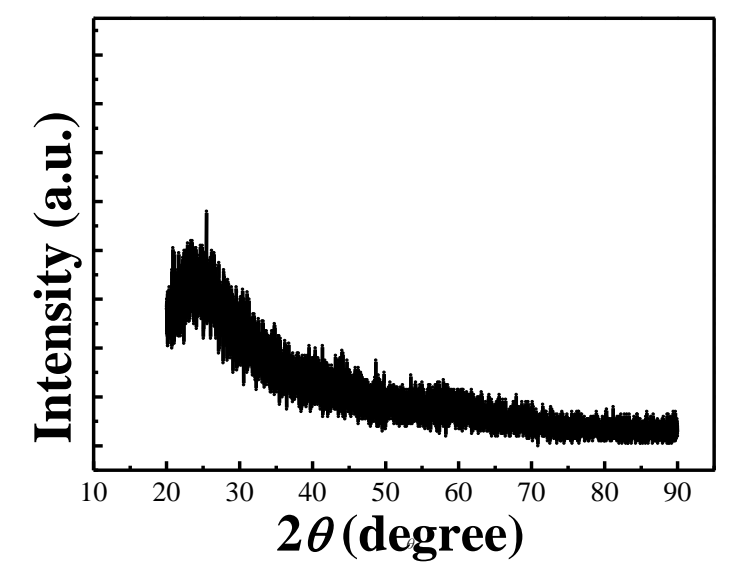

Figure 4. XRD pattern of MZN NR/glass thin films.

The bipolar resistance switching characteristics of the fabricated Al/MZN NR/ITO RRAM were compared with the device without NRs (Figure 5). Low-temperature hydrothermal method can offer sufficient oxygen vacancy [19]. For the NR-based RRAM, the set voltage is $-1.3 \mathrm{~V}$ and the reset voltage 
is $2.2 \mathrm{~V}$, which is smaller than those in devices without NRs. By sweeping a negative bias from zero to over the set voltage, the device switched from the high resistance state (HRS) to the low resistance state (LRS), called "the set process". When a positive reset voltage was applied, the device could return to HRS, which is the "reset process". Compared with MZN-based RRAM, the ON/OFF ratio (read at 0.1 V) of the Al/MZN NR/ITO device could be increased from $10^{4}$ to $10^{6}$.
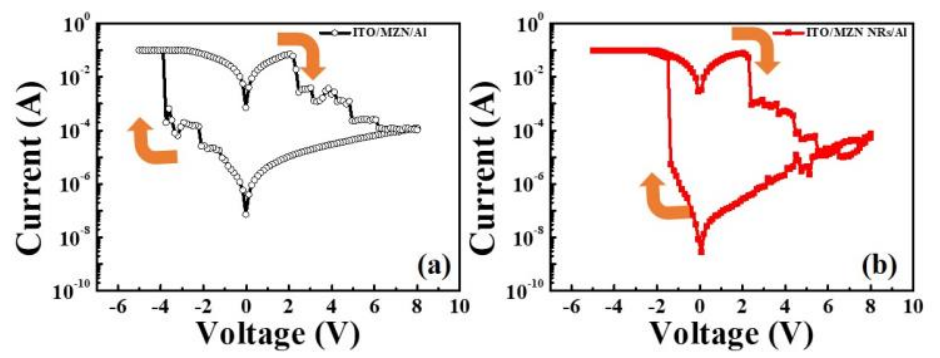

Figure 5. Typical $I-V$ curves of: (a) Al/MZN thin film/ITO structure; and (b) Al/MZN NR/ITO structure.

Figure 6 shows the logarithmic plots of $I-V$ curves for positive and negative voltage sweep regions of (Figure 6a) Al/MZN/ITO and (Figure 6b) Al/MZN NR/ITO devices, respectively. The $\log I-\log V$ curves in the HRS of Al/MZN/ITO and Al/MZN NRs/ITO devices at low voltage have a linear region with a slope of 1.01, thus implying the ohmic conduction behavior of both devices. In the high-voltage region, these slopes are related with Child's law $\left(\mathrm{I} \alpha \mathrm{V}^{2}\right)$, presenting a space charge limited conduction (SCLC). RRAM devices without and with NRs were dominated by space-charge-limited current (SCLC) in the HRS [20]. By contrast, the relationship between $\log I$ and $\log V$ in the LRS showed linear dependence, corresponding to ohmic conduction behavior. This finding implies that resistive switching mechanisms could be considered as formation and rupture of conducting filament paths.
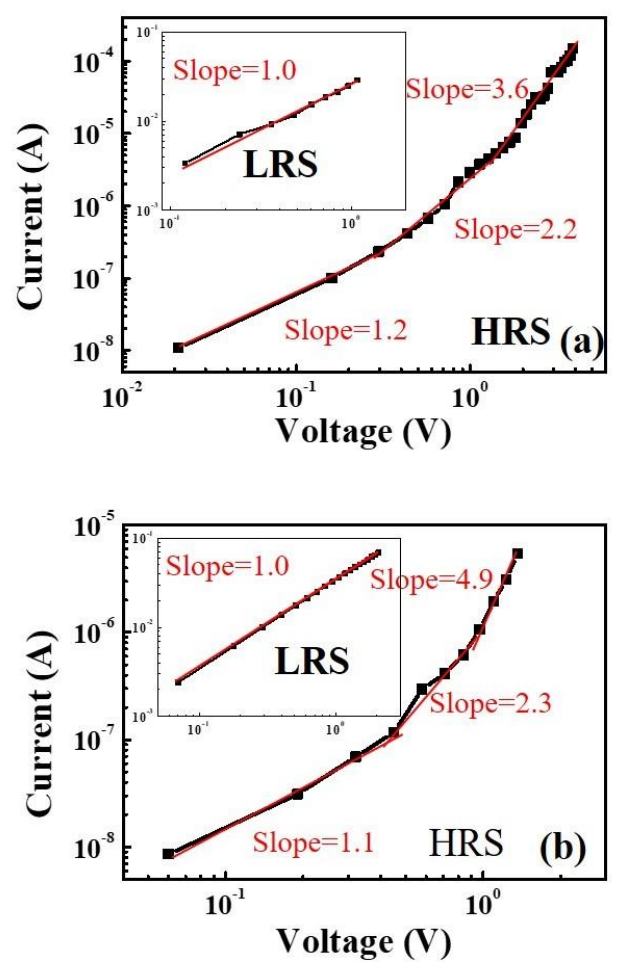

Figure 6. $\log I-\log V$ curves for the positive voltage regions and the negative voltage regions of: (a) $\mathrm{Al} / \mathrm{MZN} / \mathrm{ITO}$ device; and (b) Al/MZN NR/ITO device. The inset image shows the Log IV curves for the LRS. 
Figure 7 shows the plots the scaling trend of the LRS current versus the cell area of Al/MZN/ITO and Al/MZN NR/ITO devices. The LRS current showed a cell size-independent behavior. LRS current as a filamentary conduction current only had a slight dependence on cell area [21]. This result indicates that resistive switching behavior was related to and occurred in conductive filaments.

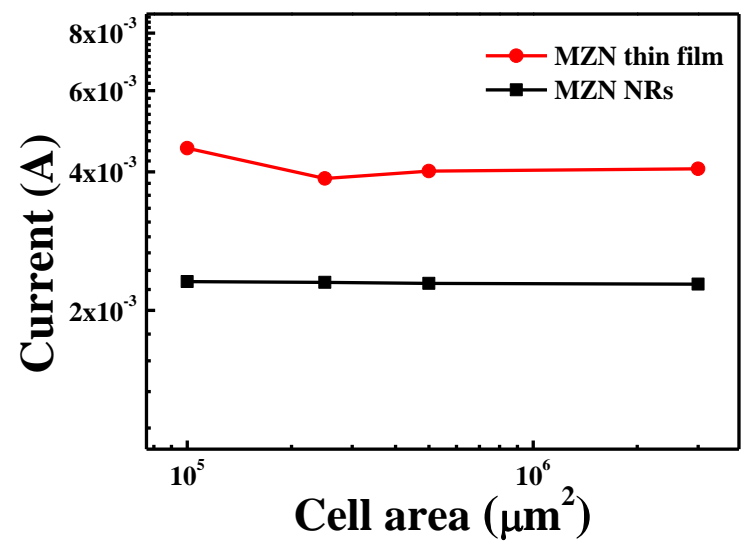

Figure 7. LRS currents versus cell areas of $\mathrm{Al} / \mathrm{MZN} / \mathrm{ITO}$ and $\mathrm{Al} / \mathrm{MZN} \mathrm{NR} / \mathrm{ITO}$ devices.

Figure 8 shows the endurance switching cycles of (Figure $8 \mathrm{a}$ ) Al/MZN/ITO and (Figure 8b) $\mathrm{Al} / \mathrm{MZN}$ NR/ITO structures, respectively, under DC voltage sweep. Figure 8 a shows the Al/MZN/ITO structure can maintain the ON/OFF ratio of more than $10^{4}$ read at $0.1 \mathrm{~V}$ after 20 continuous DC voltage switching cycles. Figure $8 \mathrm{~b}$ shows the switching endurance of Al/MZN NR/ITO memory cell over 50 switching cycles with ON/OFF ratio was approximately $10^{5}$ [22].
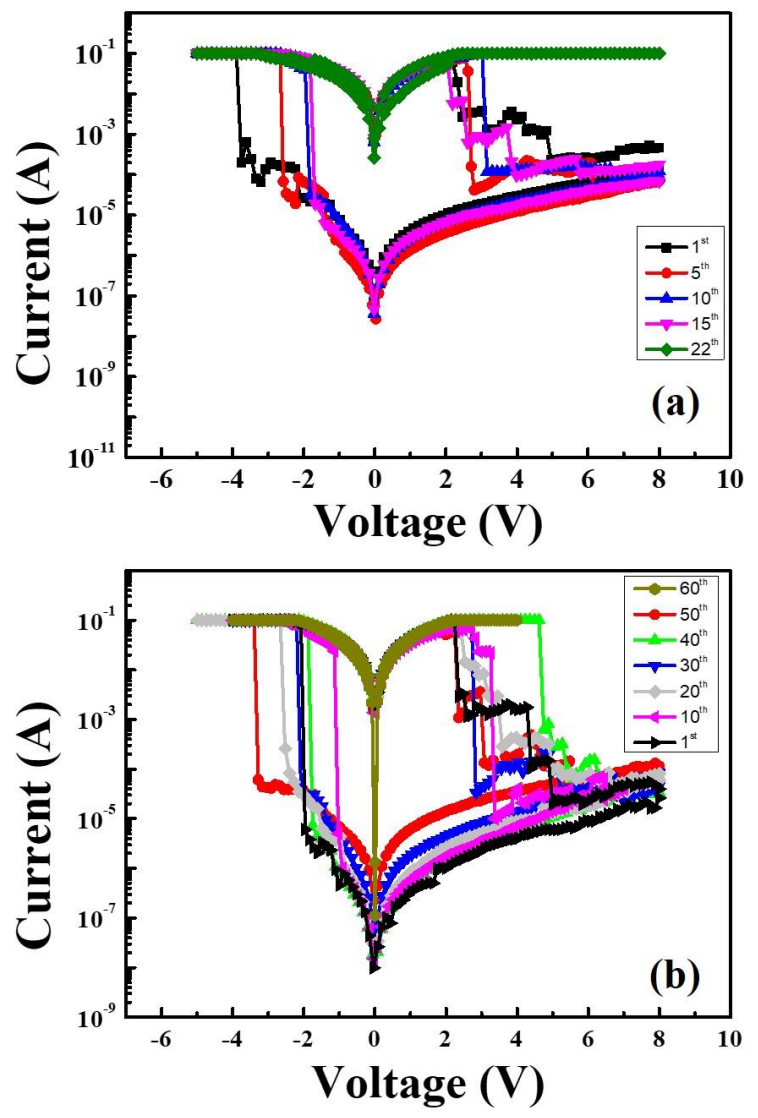

Figure 8. Switching cycles of: (a) Al/MZN/ITO device; and (b) Al/MZN NR/ITO device. 
Figure 9 shows the $\mathrm{V}_{\text {set }} / \mathrm{V}_{\text {reset }}$ distributions of (Figure 9a) Al/MZN/ITO and (Figure 9b) Al/MZN NR/ITO devices. This uniformity is better than that of Al/MZN/ITO devices (Figure 9a), whose set voltage was distributed from -5 to $-0.5 \mathrm{~V}$ and their reset voltage was distributed from 2 to $7 \mathrm{~V}$. Figure $9 \mathrm{~b}$ shows the $\mathrm{V}_{\text {set }}$ of $\mathrm{Al} / \mathrm{MZN}$ NR/ITO devices was distributed within -5 to $-0.5 \mathrm{~V}$, and $\mathrm{V}_{\text {reset }}$ was distributed within $1.5-5 \mathrm{~V}$ when the devices switched from the $\mathrm{ON}$ to the OFF state.
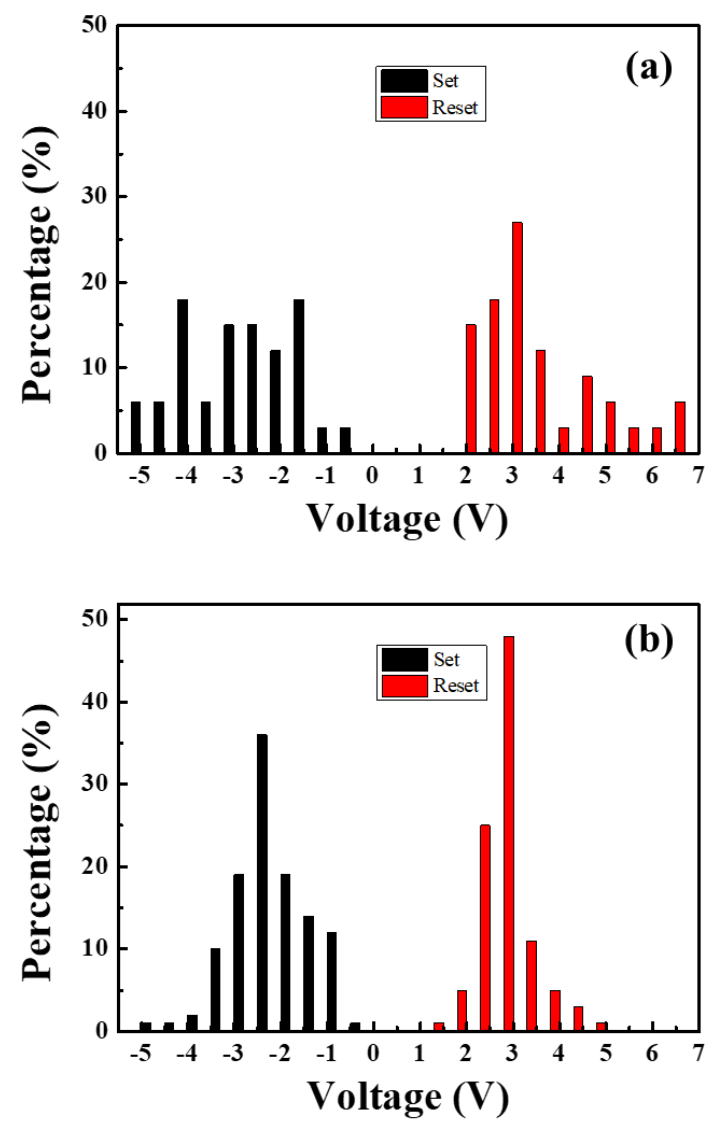

Figure 9. Set and reset voltage distributions of: (a) Al/MZN/ITO/glass device; and (b) Al/MZN NR/ITO/glass device.

Figure 10a,b presents the current distributions of $\mathrm{Al} / \mathrm{MZN} / \mathrm{ITO}$ and $\mathrm{Al} / \mathrm{MZN}$ NR/ITO devices, respectively. CV was used to evaluate the distribution. The CVs of the HRS and LRS currents of Al/MZN NR/ITO devices were $96 \%$ and $52 \%$, respectively. The CVs of HRS and LRS currents in the MZN-based RRAM devices were 50\% and 30\%, respectively [23]. Furthermore, LRS and HRS were distributed more uniformly after adding NRs, and the ON/OFF ratio was maintained at approximately $10^{5}$. The MZN NRs can concentrate oxygen vacancies on the surface of MZN NR, instead of distributing oxygen vacancies in the smooth MZN thin film. Therefore, MZN NRs can improve the uniformity of conductive filaments and thus reduce the CV.

In this study, we supposed that ionized oxygen vacancies charge and discharge electrons to achieve cycles of the formation and rupture of conductive filaments on the surface of MZN NRs according to the conduction mechanism $[8,9,24,25]$. Figure 11a shows that the injected electrons were trapped on the NR sidewall with uniform distribution of ionized oxygen vacancies. As shown in Figure 11b, the injected electrons fully occupied the trap level when the applied voltage was gradually increased to the set voltage, and subsequent electrons could transport to the ITO without being trapped. Conductive filaments are bridged on the NR sidewall. Figure 11c shows that, under negative bias, the captured electrons were released to the $\mathrm{Al}$ due to the negative electric field, resulting in an increase in the resistance. This phenomenon may explain the rupture of conductive filaments and the reset process. 


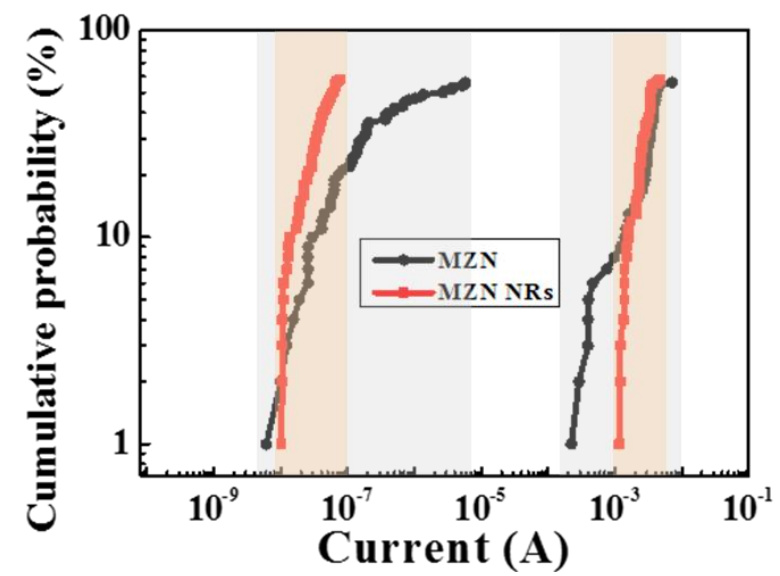

Figure 10. Current statistical distributions of: (a) Al/MZN/ITO device; and (b) Al/MZN NR/ITO device.

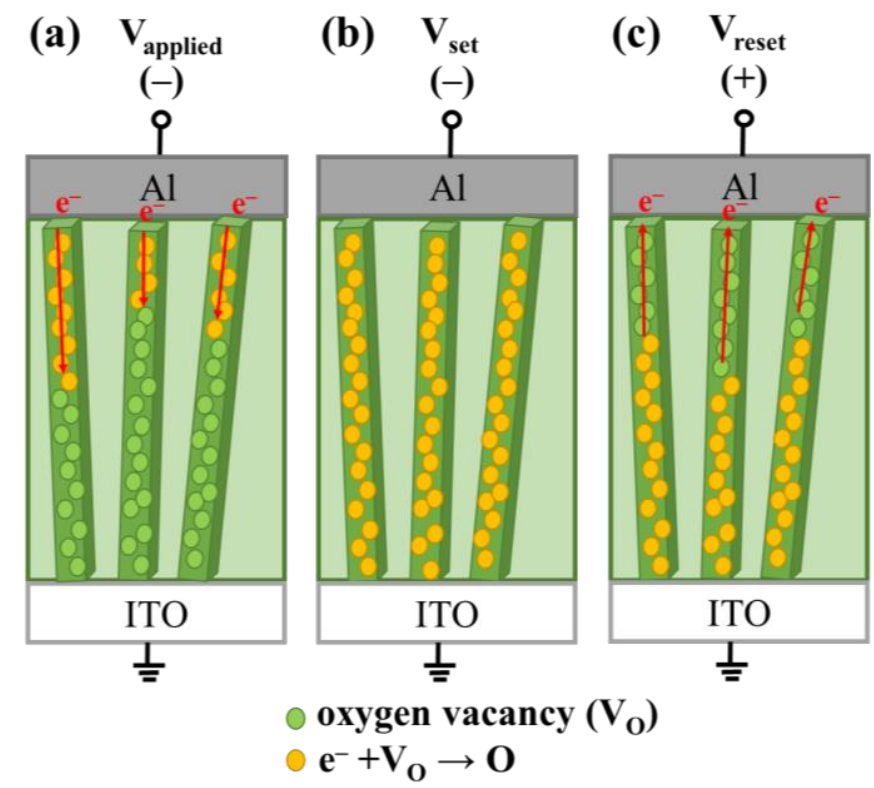

Figure 11. Schematic of the mechanisms of MZN NRs in terms of: (a) distribution of oxygen vacancies on the NR sidewall; (b) conductive filaments; and (c) rupture of conductive filaments.

Figure 12 shows the ON/OFF ratio variation in Al/MZN NR/ITO devices with increasing temperature, in which the current was measured at $0.1 \mathrm{~V}$. The ON/OFF ratio measured at room temperature was approximately $10^{7}$, and the HRS current was $4.9 \times 10^{-11} \mathrm{~A}$. When the temperature was increased from 40 to $85^{\circ} \mathrm{C}$, the HRS current increased to around $10^{-7} \mathrm{~A}$, and the ON/OFF ratio decreased to $10^{4}$ and kept the memory window without significant degradation. The result demonstrates the steady resistive switching operation of Al/MZN NR/ITO devices with temperature variation.

Figure 13 presents the retention time of Al/MZN NR/ITO RRAM devices measured at room temperature and at $85^{\circ} \mathrm{C}$. The ON/OFF ratio was maintained to at least $10^{6}$ and $10^{5}$ at room temperature and at $85^{\circ} \mathrm{C}$, respectively. The HRS and LRS were read at $0.1 \mathrm{~V}$ for over $10^{4} \mathrm{~s}$. Hence, good retention of data could be obtained, clearly revealing the nonvolatile characteristics of RRAM devices. 


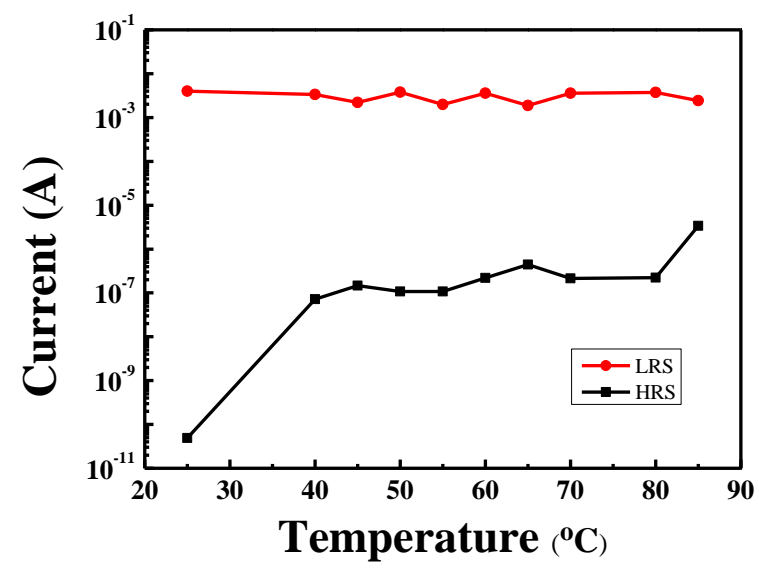

Figure 12. The typical resistive switching current-voltage curve of Al/MZN NR/ITO RRAM device at different environmental temperatures.

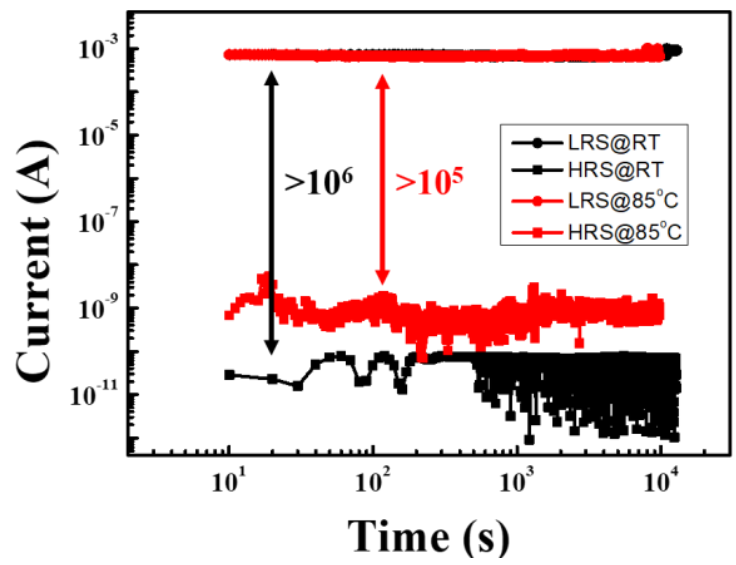

Figure 13. Data retention characteristics of Al/MZN NR/ITO RRAM devices measured at $0.1 \mathrm{~V}$.

\section{Materials and Methods}

MZN solution $(0.5 \mathrm{M})$ was prepared as follows. Magnesium acetate, zirconium n-propoxide, and nickel II acetylacetone were synthesized according to the flow chart shown in Figure 14a. An appropriate amount of magnesium acetate was added to glacial acetic acid to obtain Solution A. Zirconium n-propoxide was dissolved into 2-methoxythanol and added with nickel (II) acetylacetone and acetylacetone to produce Solution B. Solutions A and B were mixed. MZN NR film was synthesized through hydrothermal method. The MZN thin film was deposited by spin coating onto the ITO/glass substrate to act as a NR nucleation layer and baked at $100{ }^{\circ} \mathrm{C}$ for $10 \mathrm{~min}$. The MZN NR film formed an MZN NR nucleation layer in $1 \mathrm{M}$ MZN solution at $60{ }^{\circ} \mathrm{C}$ for $23 \mathrm{~h}$. After the growth of the MZN NRs, Al top electrodes were deposited with $3 \mathrm{~mm}^{2}$ patterned shadow mask by using direct current (DC) magnetron sputtering. Figure 14b shows the schematic of the MZN NR-based RRAM. 
(a)

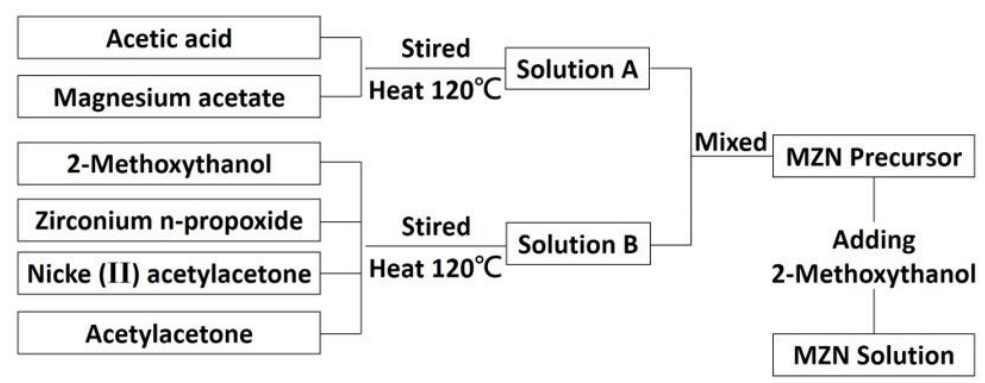

(b)

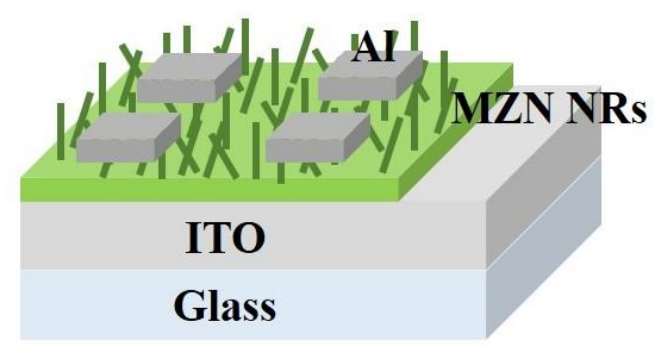

Figure 14. (a) Flow chart for preparation of 0.5 M MZN solution; and (b) schematic of Al/MZN NR/ITO device and set up for measurement.

\section{Conclusions}

MZN NRs were grown on ITO/glass substrate through hydrothermal method. Compared with its counterpart MTN thin film, MZN NR RRAM can significantly enhance the uniformity and the ON/OFF ratio from $10^{4}$ to $10^{6}$ without any forming processes. In addition, the CVs of MZN NR memory devices were $29 \%$ for ILRS and $46 \%$ for IHRS, which are lower than those of MZN film memory device (52\% for ILRS and $97 \%$ for IHRS). This result indicates that MZN NRs improved device uniformity. The electrical conduction mechanism exhibited a trap-controlled space-charge limited current. Ionized oxygen vacancies distributed on the surface of MZN NRs may lead to more uniform switching distribution. When applying the set voltage, the electrons were trapped on the NR sidewall and conductive filaments were formed, which set the device to LRS. When applying the reset voltage, the captured electrons were released and the device was reset to HRS.

Author Contributions: T.-H.S. was responsible for the device preparation and characterization and data analysis. L.-W.W. was also responsible for the material/device preparation and discussion. K.-J.L. was responsible for the modeling discussion and writing and editing the manuscript. Y.-C.C. was also responsible for the material preparation and discussion. Y.-H.W. was the advisor who monitored the progress and paper editing. All authors have read and agreed to the published version of the manuscript.

Funding: This work was supported in part by the Ministry of Science and Technology 1 of Taiwan under Grant MOST 106-2221-E-006 -219 -MY3 and MOST 108-2221-E-006 -040 -MY3

Conflicts of Interest: The authors declare no conflict of interest.

\section{References}

1. Chang, Y.C.; Xue, R.Y.; Wang, Y.H. Multilayered Barium Titanate Thin Films by Sol-Gel Method for Nonvolatile Memory Application. IEEE Trans. Electron. Devices 2014, 61, 4090-4097. [CrossRef]

2. Chang, Y.C.; Lee, K.J.; Lee, C.J.; Wang, L.W.; Wang, Y.H. Bipolar Resistive Switching Behavior in Sol-Gel MgTiNiOx Memory Device. IEEE J. Electron. Devices Soc. 2016, 4, 321-327. [CrossRef] 
3. Lee, K.J.; Chang, Y.C.; Lee, C.J.; Wang, L.W.; Wang, Y.H. Resistive Switching Characteristics in Flexible $\mathrm{Pt} / \mathrm{MZT} / \mathrm{Al}$ Memory and $\mathrm{Ni} / \mathrm{NbO}_{2} / \mathrm{Ni}$ Selector Structure. IEEE J. Electron. Devices Soc. 2018, 6, 518-524. [CrossRef]

4. Panda, D.; Tseng, T.Y. Perovskite Oxides as Resistive Switching Memories: A Review. Ferroelectrics 2014, 471, 23-64. [CrossRef]

5. Yoo, E.; Lyu, M.; Yun, J.H.; Kang, C.; Choi, Y.; Wang, L. Bifunctional resistive switching behavior in an organolead halide perovskite based $\mathrm{Ag} / \mathrm{CH}_{3} \mathrm{NH}_{3} \mathrm{PbI}_{3} \times \mathrm{Clx} / \mathrm{FTO}$ structure. J. Mater. Chem. C 2016, 4, 7824-7830. [CrossRef]

6. Balaji, V.; Senthilkumaran, S.; Thangadurai, P. Quantitative phase analysis of $\mathrm{Mg}: \mathrm{ZrO}_{2}$ nanoparticles by Rietveld refinement method. Appl. Phys. Lett. 2014, 1591, 294-295. [CrossRef]

7. Badwal, S.P.S. Zirconia-based solid electrolytes: Microstructure, stability and ionic conductivity. Solid State Lonics 1992, 52, 23-32. [CrossRef]

8. Huang, C.H.; Chou, T.S.; Huang, J.S.; Lin, S.M.; Chueh, Y.L. Self-Selecting Resistive Switching Scheme Using $\mathrm{TiO}_{2}$ Nanorod Arrays. Sci. Rep. 2017, 7, 2066. [CrossRef]

9. Chang, W.Y.; Lin, C.A.; He, J.H.; Wu, T.B. Resistive switching behaviors of ZnO nanorod layers. Appl. Phys. Lett. 2010, 96, 242109. [CrossRef]

10. Lai, Y.; Qiu, W.; Zeng, Z.; Cheng, S.; Yu, J.; Zheng, Q. Resistive Switching of Plasma-Treated Zinc Oxide Nanowires for Resistive Random Access Memory. Nanomaterials 2016, 6, 16. [CrossRef]

11. Tseng, Z.; Kao, P.C.; Shih, M.F.; Huang, H.H.; Wang, J.Y.; Chu, S.Y. Electrical bistability in hybrid ZnO nanorod/polymethylmethacrylate heterostructures. Appl. Phys. Lett. 2010, 97, 212103. [CrossRef]

12. Huang, C.Y.; Ho, Y.T.; Hung, C.J.; Tseng, T.Y. Compact Ga-Doped ZnO Nanorod Thin Film for Making High-Performance Transparent Resistive Switching Memory. IEEE Trans. Electron. Devices 2014, 6, 3435-3441. [CrossRef]

13. Huang, Y.C.; Cheng, P.Y.; Huang, K.F.; Chuang, T.C.; Lin, H.H.; Chin, T.S.; Liu, R.S.; Lan, Y.W.; Chen, C.D.; Lai, C.H. Using binary resistors to achieve multilevel resistive switching in multilayer $\mathrm{NiO} / \mathrm{Pt}$ nanowire arrays. NPG Asia Mater. 2014, 6, 81. [CrossRef]

14. Cagli, C.; Nardi, F.; Harteneck, B.; Tan, Z.; Zhang, Y.; Ielmini, D. Resistive-Switching Crossbar Memory Based on Ni-NiO Core-Shell Nanowires. Small 2011, 7, 2899-2905. [CrossRef]

15. Polsongkram, D.; Chamninok, P.; Pukird, S.; Chow, L.; Lupan, O.; Chai, G.; Khallaf, H.; Park, S.; Schulte, A. Effect of synthesis conditions on the growth of ZnO nanorods via hydrothermal method. Physica B 2008, 403, 3713-3717. [CrossRef]

16. Shen, G.H.; Tandio, A.R.; Lin, M.Y.; Lin, G.F.; Chen, K.H.; Hong, F.C.N. Low switching-threshold-voltage zinc oxide nanowire array resistive random access memory. Thin Solid Film. 2016, 618, 90-94. [CrossRef]

17. Zhao, Y.; Jin, J.; Yang, X. Hydrothermal synthesis of titanate nanowire arrays. Mater. Lett. 2007, 61, $384-388$. [CrossRef]

18. Wang, Z.; Xu, H.; Li, X.; Zhang, X.; Liu, Y.; Liu, Y. Flexible resistive switching memory device based on amorphous InGaZnO film with excellent mechanical endurance. IEEE Electron. Device Lett. 2011, 32, 1442-1444. [CrossRef]

19. Chu, D.; Younis, A.; Li, S. Direct growth of $\mathrm{TiO}_{2}$ nanotubes on transparent substrates and their resistive switching characteristics. J. Phys. D Appl. Phys. Lett. 2012, 45, 355306. [CrossRef]

20. Lampert, M.A. Simplified theory of space-charge-limited currents in an insulator with traps. Phys. Rev. 1956, 103, 1648. [CrossRef]

21. Chen, F.T.; Lee, H.Y.; Chen, Y.S.; Chen, P.S.; Gu, P.; Chen, C.W.; Hsu, Y.Y.; Liu, W.H.; Chen, W.S.; Tsai, M.J.; et al. Size dependence of TiN/ $\mathrm{HfO}_{2} / \mathrm{Ti}$ MIM ReRAM resistance states: Model and experimental results. Curr. Appl. Phys. 2010, 10, 75-78. [CrossRef]

22. Kim, A.; Song, K.; Kim, Y.; Moon, J. All solution-processed, fully transparent resistive memory devices. ACS Appl. Mater. Interfaces 2011, 3, 4525-4530. [CrossRef] [PubMed]

23. Lee, W.; Yoon, S. Effects of device size and material on the bending performance of resistive-switching memory devices fabricated on flexible substrates. Appl. Phys. Lett. 2017, 110, 192103. [CrossRef] 
24. Hong, Y.S.; Chen, J.Y.; Huang, C.W.; Chiu, C.H.; Huang, Y.T.; Huang, T.K.; He, R.S.; Wu, W.W. Single-crystalline $\mathrm{CuO}$ nanowires for resistive random access memory applications. Appl. Phys. Lett. 2015, 106, 173103. [CrossRef]

25. Wang, T.Y.; Meng, J.L.; He, Z.Y.; Chen, L.; Zhu, H.; Sun, Q.Q.; Ding, S.J.; Zhang, D.W. Atomic Layer Deposited $\mathrm{Hf}_{0.5} \mathrm{Zr}_{0.5} \mathrm{O}_{2}$-based Flexible Memristor with Short/Long-Term Synaptic Plasticity. Nanoscale Res. Lett. 2019, 14, 102. [CrossRef]

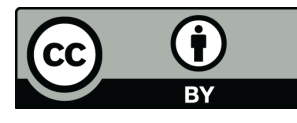

(C) 2020 by the authors. Licensee MDPI, Basel, Switzerland. This article is an open access article distributed under the terms and conditions of the Creative Commons Attribution (CC BY) license (http://creativecommons.org/licenses/by/4.0/). 